\title{
Explicit \& Implicit Interaction Design for Multi-Focus Visualizations
}

Simon Butscher

HCI Group, University of Konstanz

Universitätsstraße 10

Konstanz, 78457, Germany

Simon.Butscher@uni-konstanz.de

\section{Abstract}

Many tasks that have to be performed to analyze data in large visual information spaces require the user to have several foci. This is for example the case for comparing or organizing digital artefacts. In my research, I explore alternative interaction concepts for multi-focus visualizations in the context of single and multi-user scenarios. Alongside explicit interaction for navigating within multi-focus visualizations I investigate implicit interaction for making the visualization react on the social and spatial context. To evaluate different designs, measures like task completion time, spatial memory and subjective preferences are examined.

Permission to make digital or hard copies of part or all of this work for personal or classroom use is granted without fee provided that copies are not made or distributed for profit or commercial advantage and that copies bear this notice and the full citation on the first page. Copyrights for thirdparty components of this work must be honored. For all other uses, contact the Owner/Author. Copyright is held by the owner/author(s).

\author{
Author Keywords \\ Multi-Focus+context; implicit interaction; explicit \\ interaction; touch interaction; proxemic interactions
}

\section{ACM Classification Keywords}

H.5.2. Information interfaces and presentation (e.g., $\mathrm{HCI}$ ): User Interfaces - Interaction styles

\section{Research Background}

I received a degree (german Diplom) in Business Information Systems at the University of Cooperative Education in Ravensburg, Germany. For my thesis I designed and implemented a solution for effective data analysis within a business intelligence application for companies in the healthcare sector. In addition I hold a master's degree in Information Engineering from the University of Konstanz, Germany. For my master's thesis I designed, implemented, and evaluated a focus+context visualization and remote interaction concept for the analysis of the traffic situation on a road network [13]. With this project, I gained valuable expertise and insights into the combination of focus+context visualizations with suitable input modalities for their control. Since the project's completion, I have been interested in the interplay between visualization techniques and input modalities and especially in the combination of multi-focus 


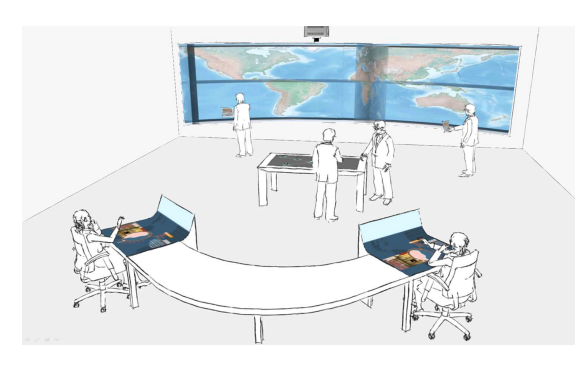

Figure 1. The social and spatial context within a future control room.

Relationships of people to each other and to the displays can be used for an implicit interaction with visualizations. visualizations with alternative input modalities to

facilitate data analysis tasks in visual information spaces.

Since the beginning of my Ph.D. I have been affiliated with different projects ranging from designing future control rooms, ${ }^{1}$ blending the physical and digital space in public ${ }^{2}$ or academic ${ }^{3}$ libraries and analyzing mobile health care data. One of the main tasks to be supported within all of these projects is data analysis. For my future research I will focus on two application scenarios: the analysis of traffic situation in traffic control rooms and the analysis of health data collected with mobile devices. My supervisor is Harald Reiterer from the University of Konstanz.

\section{Motivation}

Many tasks that have to be performed in multiscale visual information spaces require several foci. This is also true for data analysis tasks in which digital artifacts have to be compared or organized. Whereas virtually all interfaces allow the user to change between foci over time (e.g., by navigating a map with pan/zoom gestures), fewer interfaces allow the simultaneous presentation of multiple foci. Examples of such multi-focus visualization techniques include multi-window systems, split-screen interfaces, and a variety of research prototypes (e.g. $[5,6,11]$ ).

Multi-focus visualization techniques are a well-studied field in $\mathrm{HCI}$ (for an overview of research about multifocus visualizations see [4]). Yet, research on how alternative input modalities can facilitate the interaction

\footnotetext{
${ }^{1}$ http://hci.uni-konstanz.de/HolisticWorkspace

${ }^{2}$ http://hci.uni-konstanz.de/Libros

${ }^{3}$ http://hci.uni-konstanz.de/BlendedLibrary
}

with these visualizations is limited. Novel input modalities offer great potential to design interaction concepts which suit multi-focus visualizations better than state-of-the art approaches. For example, in contrast to many other input devices, multi-touch surfaces seem to naturally enable multi-focus interaction, e.g., by using one hand per focus point. Furthermore and with respect to multi-user scenarios, many alternative input modalities (e.g. touch or gestural input) facilitate a democratized form of collaboration, which mouse input, by contrast, does not.

However explicit interaction to navigate within a multifocus visualization is only one part of the possible design space. The interpretation of the social and spatial context (see Figure 1) can be another part and is especially promising for an implicit interaction to automatically adapt the appearance of the visualization (e.g. placing information on a display depending on the position of a user in front of it).

Consequently, I intend to support the different activities of data analyst, such as navigating in large information spaces or comparing digital artifacts by using multi-focus visualization techniques that exploit alternative input modalities and combine explicit and implicit interaction styles.

\section{Background and Related Work}

Although multi-focus visualization techniques are a well-studied research topic, an unanswered question is how these visualization techniques can benefit from alternative input modalities. Most research has concentrated on the presentation, not on the interaction (e.g. [5]). Only some research has emphasized the interaction with the visualization and 

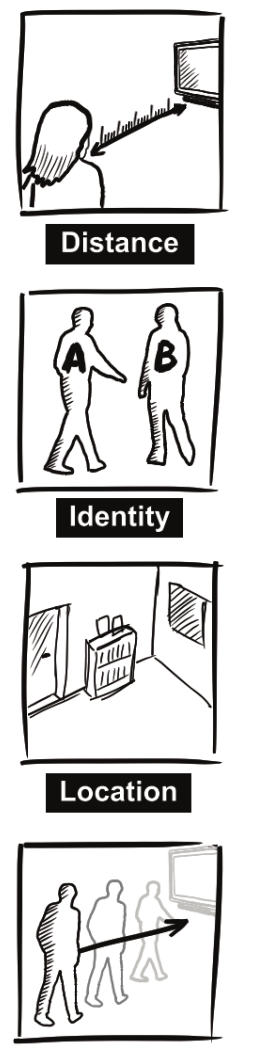

Movement

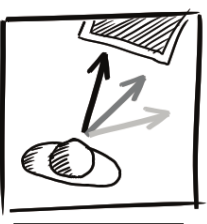

Orientation

Figure 2. The five dimensions of proxemics [7]. makes use of input modalities like touch to facilitate the definition of areas of interest within a visual information space (e.g. $[8,11])$. However, they fail to exploit the additional possibilities provided by alternative input modalities in all facets.

Most research in the field of multi-focus visualizations is limited to explicit interaction and ignores the possibilities of adapting the visualizations to the implicit input of the user. Implicit interaction can be realized by analyzing and interpreting the spatial and social context. Some research areas other than multi-focus visualizations have implemented systems that provide a mixture of explicit interaction and implicit interaction. An interactive public display installation for example interprets the spatial context (position of the user in front of a display) and thus shows customized information to the user [14]. Another system designed for multi-display environments uses the users head position as implicit input to correct the perspective distortion of the elements visualized on the screens [12].

Proxemic Interactions [7], provide a general perspective on the spatial and social context in terms of relationships between persons and objects (e.g. displays) but also between persons to each other. To describe these relationships five dimensions of proximity are proposed: distance, orientation, movement, identity, and location (see Figure 2). These proxemic dimensions offer a design space for adaptive visualizations which react on the spatial and social environment.

\section{Research Approach}

I make use of a taxonomy to describe the design space for investigating data analysis tasks. This taxonomy is built on the task to perform and the number of parallel users:

Tasks: In my research I focus on data analysis in visual information spaces in the sense of exploring the space, searching for objects, comparing objects and organizing them. Depending on the task, different requirements are relevant, like orientation within the information space, spatial memory to remember object locations or multiple foci to compare or move digital artefacts to other location in the visual space.

Number of parallel users: Data analysis can be an individual but also a collaborative activity, e.g., within a control room. For collaborative scenarios I have to deal with additional requirements like a democratized from of collaboration or an enhanced situational awareness (awareness of ongoing activities, and awareness of who is in control of shared artefacts [9]).

To investigate how multi-focus techniques can facilitate data analysis tasks different combinations of the two dimensions of this taxonomy have to be explored. Depending on the task and the number of parallel users, either single-focus or multi-focus solutions may be appropriate. Whereas the visualization has to support multiple foci for multiple users regardless of the task at hand, in single user scenarios only the compare task requires multiple foci. The combination of the task and the number of parallel users also influences the choice of the input modality.

Proxemic dimensions can help to capture the spatial and social context and enable an automatic reaction (e.g. if two persons move closer to each other the focus 




Figure 3. Supporting single users comparing human neural stem cells: (left) SpaceFold - Fold the visual space like a sheet of paper to bring two areas of interest closer to each other; (right) PhysicLenses Create multiple magnification lenses to see detailed views of areas of interest.

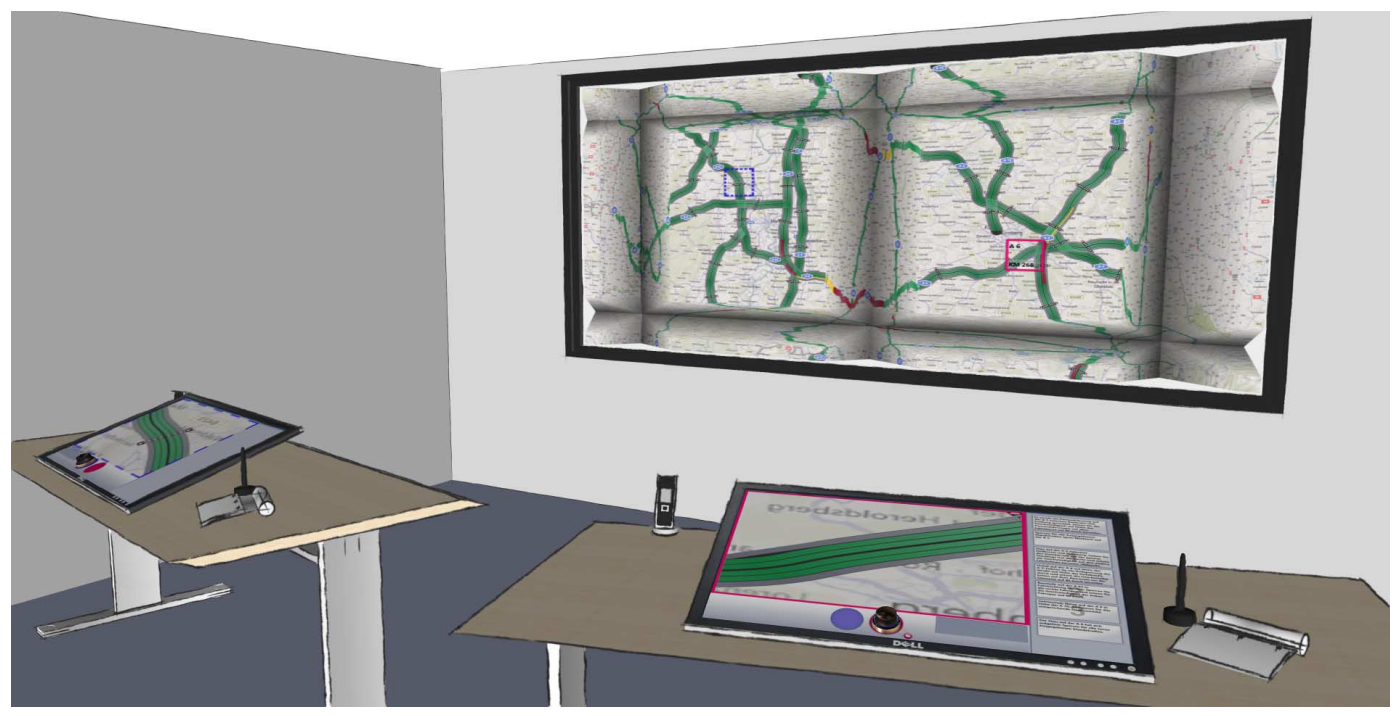

Figure 4. Supporting multiple users analyzing the traffic situation: A multi-focus visualization on a large wall-sized display can be controlled remotely by the operators through self-centering devices. of both users could be merged in order to facilitate discussions about digital artefacts). Thus in multi-user ecosystems proxemic dimensions plays a key role in analyzing the social context, and can be used to consider social conventions. Whereas I already

investigated some combinations of the taxonomy other combinations are still to be explored.

\section{Completed Work}

For the dimension of single-user interaction to perform compare and organize (drag \& drop) tasks we conducted a controlled experiment to compare different solutions [1]. We introduced two novel navigation techniques that combine multiple foci and bimanual touch, and thus enable the isochronic definition of areas of interest, leading to simultaneous multifocus navigation. SpaceFold folds the visual space in the third dimension, allowing users to bring objects closer to each other (see Figure 3, left). Our technique enables a direct, bimanual manipulation of a folded space and is highly flexible. PhysicLenses uses multiple magnification lenses to compare objects (see Figure 3, right). Using a physics model, PhysicLenses introduces a general solution for the arrangement of multiple lenses within the viewport. We conducted a controlled experiment with 24 participants to compare the techniques with split screen. The results show that SpaceFold significantly outperformed all other techniques, whereas PhysicLenses was just as fast as split screen.

For the multi-user scenario within a traffic control room we investigated solutions for collaboratively analyzing the traffic situation [2]. First, to gain an understanding of relevant tasks and the social environment that shape the work of operators, a context-of-use analysis in two 


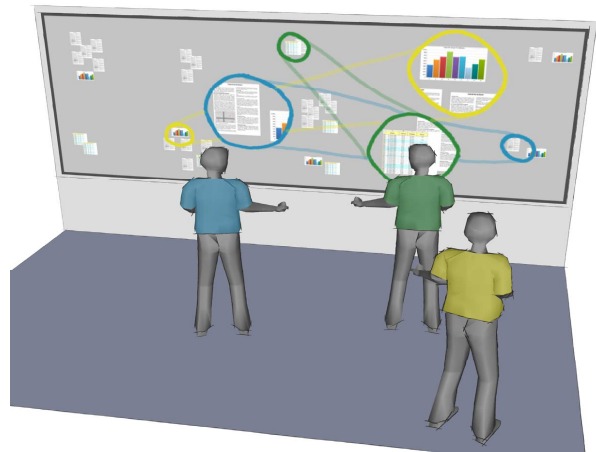

Figure 5. Supporting multiple users to analyze a visual information space: A

combination of an explicit interaction for the selection of areas of interest and an implicit interaction for the tailored positioning of the information. The position of the user in front of the display is mapped to the position were detailed information are shown. The aim is to enable seamless switching between tightly-coupled collaboration and looselycoupled parallel work.

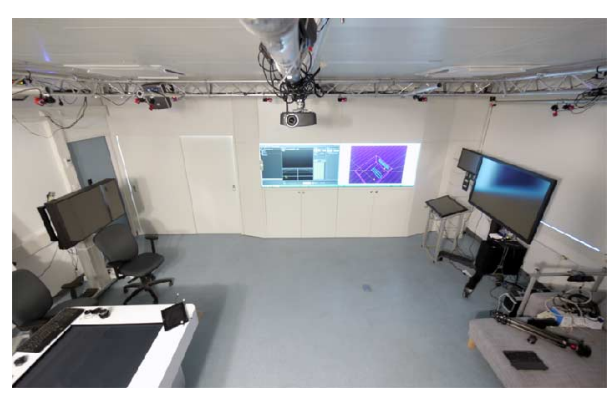

Figure 6. Multi-display environment equipped with OptiTrack tracking system to capture the spatial and social context. freeway traffic monitoring control rooms was conducted. Based on the analysis we created a multifocus visualization for large wall-sized displays, which makes it possible to examine local details without losing contextual information (see Figure 4). We combined a visualization based on the SpaceFolding technique by Elmqvist et al. [5] with our content-aware navigation technique [13]. To evaluate the applicability of the concept, a study with eleven participants from the domain of traffic control rooms was conducted. The results show that the multi-focus visualization

facilitated the awareness on ongoing activities. It enabled an implicit communication, which helps the operators to coordinate themselves.

\section{Future Work}

My future research will focus on data analysis in multiuser environments. Proxemic Interactions are used to analyze and interpret the spatial and social context. I am especially interested not only in considering the distance and orientation of people to a display but also to each other. Furthermore the movement of people can give insight to the actual situation. Possible interpretations of these metrics could be that if people move closer to the display they want to see the information space in greater detail, or that if people move closer to each other they want to discuss about a shared artifact. As users switch between individual tasks and collaborative tasks, switching between several independent foci on the information space and shared foci is an issue which needs to be investigated.

Proxemic measures can either be used as explicit input (e.g. defining a focus region of a multi-focus

visualization according to a user's location and orientation in physical space [3]) or as implicit input modality to adapt visualizations. Figure 5 shows a concept in which we combine explicit and implicit input for the manipulation of a multi-focus visualization. The explicit input modality in form of a smartphone used as touchpad is used to define the position of a focus region. The location of the user as an implicit input modality is used to place the enlarged view of the selected focus region directly in front of the user.

In order to implement prototypes and evaluate them to show the feasibility of multi-focus visualizations using proxemic dimensions I make use of a laboratory equipped with an OptiTrack ${ }^{4}$ tracking system (see Figure 6). The tracking systems consists of 24 infrared camera which are connected to the Proximity Toolkit

[10]. This toolkit offers great support in capturing the proximic dimensions.

\section{Statement of Thesis and Goals}

In my dissertation I address the research question of how to use combinations of explicit and implicit interaction to control multi-focus visualizations for large visual information spaces. My contributions are:

1.) Explore alternative input modalities and interaction concepts for navigating multi-focus visualizations of visual information spaces to support data analysis tasks like searching, comparing or organizing digital artefacts.

2.) Design and implement prototypes that employ proxemic dimensions as an implicit input modality for adapting multi-focus visualizations depending on the spatial and social context.

${ }^{4}$ https://www.naturalpoint.com/optitrack/ 
3.) Evaluate the developed concepts and prototypes within controlled experiments. Depending on the task metrics like task completion time, mental workload, spatial memory or subjective preferences are investigated.

\section{Expected Contributions}

Through my research, I aim to develop interaction concepts to support data analysis tasks through multifocus visualization techniques and combine them with alternative input modalities to support explicit and implicit interaction. To show the feasibility of the concepts for real world problems I will apply the concepts to single-user and multi-user scenarios from two different domains.

\section{References}

[1] Butscher, S., Hornbæk, K., and Reiterer, H. SpaceFold and PhysicLenses: Simultaneous Multifocus Navigation on Touch Surfaces. In Proc. AVI 2014, ACM Press (2014), 209-216.

[2] Butscher, S., Müller, J., Schwarz, T., and Reiterer, $\mathrm{H}$. Blended Interaction as an Approach for Holistic Control Room Design. CHI 2013 Workshop on Blended Interaction, (2013).

[3] Butscher, S., Müller, J., Weiler, A., Rädle, R., Reiterer, H., and Scholl, M. Multi-user Twitter Analysis for Crisis Room Environments. Collaborative HumanComputer-Interaction with Big Wall Displays BigWallHCI 2013, 3rd JRC ECML Crisis Management Technology Workshop, Publications Office of the European Union (2013), 28-34.

[4] Cockburn, A., Karlson, A., and Bederson, B.B. A Review of Overview+Detail, Zooming, and

Focus+Context Interfaces. ACM Computing Surveys (CSUR) 41, 1 (2008).
[5] Elmqvist, N., Henry, N., Riche, Y., and Fekete, J.D. Mélange: Space Folding for Multi-Focus Interaction. In Proc. CHI 2008, ACM Press (2008), 1333-1342.

[6] Forlines, C. and Shen, C. DTLens: Multi-user Tabletop Spatial Data Exploration. In Proc. UIST 2005, ACM Press (2005), 119-122.

[7] Greenberg, S., Marquardt, N., Ballendat, T., DiazMarino, R., and Wang, M. Proxemic Interactions: The New Ubicomp ? interactions 18, January (2011), 42-50.

[8] Käser, D., Agrawala, M., and Pauly, M.

FingerGlass: Efficient Multiscale Interaction on

Multitouch Screens. In Proc. CHI 2011, ACM Press (2011), 1601-1610.

[9] Kulyk, O., van der Veer, G., and van Dijk, B.

Situational awareness support to enhance teamwork in collaborative environments. In Proc. ECCE 2008, ACM Press (2008), 18-22.

[10] Marquardt, N., Diaz-Marino, R., Boring, S., and Greenberg, S. The Proximity Toolkit: Prototyping Proxemic Interactions in Ubiquitous Computing Ecologies. In Proc. UIST 2011, ACM Press (2011), 315325.

[11] Mikulecky, K., Hancock, M., Brosz, J., and Carpendale, S. Exploring Physical Information Cloth on a Multitouch Table. In Proc. ITS 2011, ACM Press (2011), 140-149.

[12] Nacenta, M.A., Sakurai, S., Yamaguchi, T., et al. Econic: a Perspective-Aware Interface for Multi-Display Environments. In Proc. UIST 2007, ACM Press (2007), 279-288.

[13] Schwarz, T., Butscher, S., Müller, J., and Reiterer, $\mathrm{H}$. Content-aware navigation for large displays in context of traffic control rooms. In Proc. AVI 2012, ACM Press (2012), 249-252.

[14] Vogel, D. and Balakrishnan, R. Interactive Public Ambient Displays: Transitioning from Implicit to

Explicit, Public to Personal, Interaction with Multiple Users. In Proc. UIST 14, ACM Press (2004), 137-146. 